\title{
Prevalence of monoclonal gammopathy of undetermined significance in a large population with annual medical check-ups in China
}

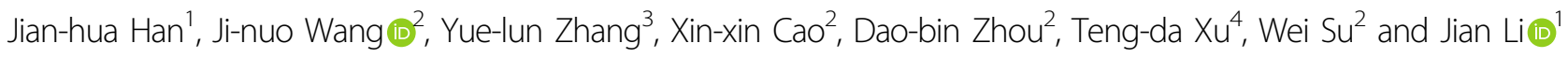

Dear Editor,

Monoclonal gammopathy of undetermined significance (MGUS) is a clinically asymptomatic premalignant plasma cell disorder, which may develop to multiple myeloma (MM), light-chain amyloidosis, Waldenström macroglobulinemia, or other related malignancies ${ }^{1}$. The crude prevalence of MGUS varies from $0.05 \%$ to $6.1 \%$ among distinct population from different countries, which is influenced by race, age, sex, family history, immunosuppression, and pesticide exposure ${ }^{2}$. So far, many population-based studies of MGUS come from the white or black population living in Western and African countries. One of the largest population-based study in the USA showed that the prevalence of MGUS was $3.2 \%$ in the Caucasian general population aged $\geq 50$ years $^{3}$, while the rate was even higher in African-Americans to be $3.7 \%$. However, data on the epidemiology of MGUS remain largely undefined in Asians. Some populationbased studies among population from Thailand, Korea, and Hong Kong were relatively limited due to small sample sizes ${ }^{5-7}$. Therefore, we conducted this study to determine the prevalence and characteristics of MGUS among a large population with annual medical check-ups in a well-defined geographic area in China.

From December 2013 to April 2019, a total of 316,616 people received annual medical check-ups at the Peking

Correspondence: Teng-da Xu (xutd@pumch.cn) or Wei Su

(suwei_64@163.com) or Jian Li (lijian@pumch.cn)

'Department of Clinical Laboratory, Peking Union Medical College Hospital,

Chinese Academy of Medical Sciences and Peking Union Medical College, Beijing, China

${ }^{2}$ Department of Hematology, Peking Union Medical College Hospital, Chinese Academy of Medical Sciences and Peking Union Medical College, Beijing,

China

Full list of author information is available at the end of the article

These authors contributed equally: Jian-hua Han, Ji-nuo Wang, Yue-lun Zhang
Union Medical College Hospital. Generally, people who received annual medical check-ups at our institute were apparently healthy and reflected a population with relatively high social economic status and health consciousness in Beijing, China. All the people with measurement of serum protein electrophoresis were included in our analysis. People were excluded if age or sex was missing in the record. The information collected at the first time when the person came to our institute was regarded as baseline data, while consecutive follow-up records of the same participant were also collected (Supplementary Fig. 1). This study was approved by the institutional ethics committee of Peking Union Medical College Hospital and informed consent was waived because there is no information that can help to identify individuals.

MGUS was defined in accordance with previous definition by International Myeloma Working Group ${ }^{8}$. Serum $\mathrm{M}$ protein was detected by capillary electrophoresis (Sebia, Parc Technologique Leonard de Vinci, Cedex, France). Patients with a positive or suspicious M protein were suggested to be referred to the hematological clinic for further assessment, including serum and/or urine immunofixation electrophoresis (IFE) to determine the type of immunoglobulin and serum free light chain (FLC) assays for quantification. Serum IFE was performed using the SPIFE ${ }^{\oplus}$ ImmunoFix-9 Kit (Helena Laboratories, TX, USA). Urine IFE was detected by the HYDRAGEL 9 IF Kit (Sebia, Parc Technologique Leonard de Vinci, Cedex, France). FLC measurements were performed by an IMMAGE 800 automated nephelometer (Beckman Coulter, CA, USA) using commercially available kits (Hevylite, The Binding Site Ltd, Birmingham, UK). Published reference ranges for $\mathrm{K}$ and $\lambda$ FLC were used (3.3-19.4 and 5.7-26.3 mg/L, respectively). An abnormal $\kappa / \lambda$ FLC ratio was defined as $<0.26$ or $>1.65^{9}$. Serum 
concentrations of immunoglobulin G (IgG), immunoglobulin $\mathrm{M}(\mathrm{IgM})$, and immunoglobulin A (IgA) were evaluated by serum immunoglobulin assay.

The overall prevalence of MGUS was calculated by the number of people with MGUS in each age and sex stratum divided by the number of subjects in that stratum who underwent a medical check-up. Descriptive statistics included medians with minimum and maximum for continuous variables and counts and percentages for categorical variables. Exact 95\% confidence intervals (CIs) for prevalence were computed using the binomial distribution. Differences between groups normally distributed with homogeneity of variance were analyzed with Student's $t$ test. All tests conducted were two tailed, and $P$ values $<0.05$ were considered statistically significant. Statistical analyses were performed with the R statistical software (Version 3.4.0) and SPSS 20.0 software (SPSS, Chicago, IL, USA).

Overall, 154,597 healthy participants from Beijing, China were finally enrolled in our study, including 71,892 females and 82,705 males (Supplementary Table 1). The average age of all participants was $45.5 \pm 12.8$ years. MGUS was diagnosed in 843 patients $(0.53 \%, 95 \% \mathrm{CI}$ $0.49-0.57 \%)$. The median age at presentation was 58 years (range, 25-96). The overall prevalence of MGUS was $1.11 \%$ (95\% CI 1.02-1.18\%) among participants aged $\geq 50$ years, $2.57 \%$ (95\% CI 2.22-2.98\%) among those aged $\geq 70$ years. In both sexes, prevalence increased with age (Fig. 1): $0.1 \%$ ( $<40$ years), $0.36 \%$ ( $40-49$ years), $0.78 \%$ (50-59 years), $1.28 \%$ (60-69 years), $2.19 \%$ (70-79 years), and $3.77 \%$ ( $\geq 80$ years) separately (Supplementary Table 1 ). MGUS was found in 543 of the 82,705 men, as compared with 280 of the 71,892 women $(0.66 \%$ vs $0.39 \%, P<0.001)$. The prevalence among men aged $\geq 50$ years was similar to that among women a decade older (Fig. 1).

The median concentration of serum $M$ protein was $1.4 \mathrm{~g} / \mathrm{L}$ (range, unmeasurable-27.8 $\mathrm{g} / \mathrm{L}$ ). The $\mathrm{M}$ protein level was too low to measure in 204 patients (24.79\%), between 0.2 and $5 \mathrm{~g} / \mathrm{L}$ for $460(55.89 \%)$ and $>15 \mathrm{~g} / \mathrm{L}$ for 16 (1.9\%) of the 843 MGUS patients (Supplementary Table 2 ). There was no significant difference in the concentration of the monoclonal protein among the age groups. Of the 602 patients who were tested for IFE, the isotype of the monoclonal immunoglobulin was $\operatorname{IgG}$ in 396 (65.78\%), IgA in 135 (22.43\%), IgM in 53 (8.80\%), IgD in 2 $(0.33 \%)$, light chain in $3(0.50 \%)$, and biclonal in 13 $(2.16 \%)$. The serum light-chain type was kappa in 305 (50.66\%) and lambda in $255(48.67 \%)$ patients, while 4 patients $(0.66 \%)$ with biclonal $\mathrm{M}$ protein had both kappa and lambda light chain. Of the 440 people who were tested for FLC, 98 patients (22.27\%) had an abnormal FLC ratio $(<0.26$ or $>1.65)$.

The concentration of uninvolved immunoglobulins was reduced in 48 of the 313 patients (15.34\%) who underwent an immunoglobulin assay. Among them, 39 (12.46\%) patients had a reduction in 1 of the 2 measured uninvolved isotypes of polyclonal immunoglobulins, and 8 (2.56\%) patients had a reduction in both isotypes. Only one patient with the light chain $\lambda$ subtype had a reduced level of three uninvolved immunoglobulins. Urine from 165 subjects with MGUS was tested by means of immunofixation electrophoresis. A monoclonal kappa light chain $(12.73 \%)$ or lambda light chain $(6.67 \%)$ was found in 32 patients. Four patients $(2.42 \%)$ had M protein detected in urine. One patient was found to have both $M$ protein and kappa light chain by urine immunofixation. IgG isotype, $M$ protein $<15 \mathrm{~g} / \mathrm{L}$, and normal FLC ratio were found in 251 patients (57.05\%), while the remaining 189 participants (42.95\%) had 1 (137, 31.14\%), 2 (51, 11.59\%), or $3(1,0.23 \%)$ abnormal risk factors. Table 1 lists the laboratory features of MGUS patients.

In the present study, we quantified the prevalence of MGUS among a relatively healthy population in the Beijing area. This is the largest screening study and population-based study of MGUS in the Chinese population so far. Our findings are consistent with previous
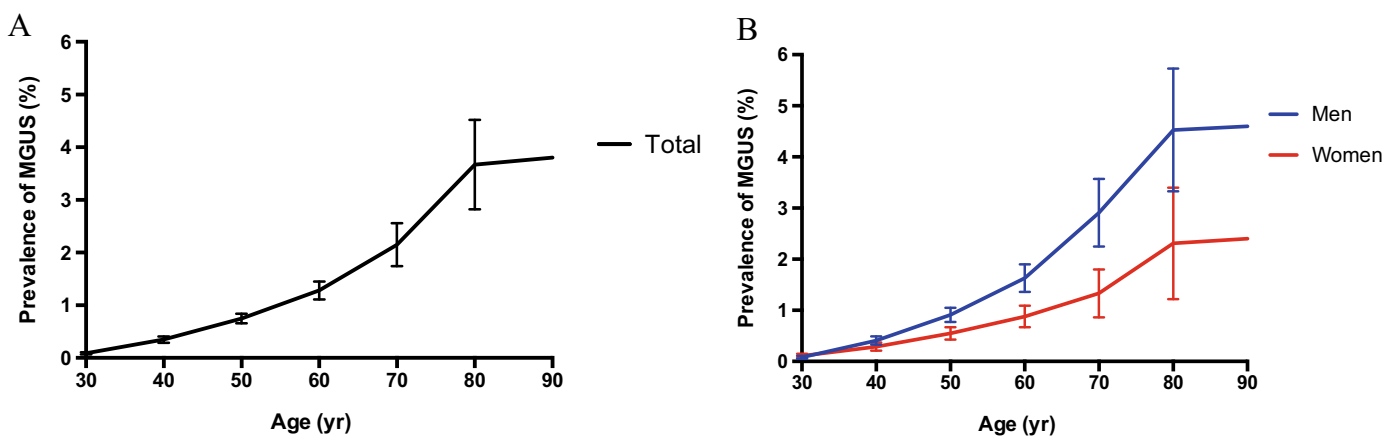

Fig. 1 Prevalence of MGUS according to age and sex groups. a Prevalence of MGUS according to age groups in the Chinese population. $\mathbf{b}$ Prevalence of MGUS according to age and sex groups in the Chinese population. The I bars represent $95 \%$ confidence intervals. Years of age $<30$ have been collapsed to 30 years of age. Years of age $>90$ have been collapsed to 90 years of age. 
Table 1 Characteristics of MGUS patients among people with annual medical check-ups in Beijing, China.

\begin{tabular}{|c|c|}
\hline Characteristics & $\begin{array}{l}\text { Patients, number/total } \\
\text { number }\left(\%^{\mathrm{a}}\right)\end{array}$ \\
\hline Monoclonal isotype (sIFE) & $602^{b}$ \\
\hline $\lg G$ & $396(65.8)$ \\
\hline $\lg A$ & $135(22.4)$ \\
\hline $\lg M$ & $53(8.8)$ \\
\hline $\lg D$ & $2(0.3)$ \\
\hline Biclonal & $13(2.2)$ \\
\hline Light chain & $3(0.5)$ \\
\hline Type of light chain (sIFE) & $602^{\mathrm{b}}$ \\
\hline k & $305(50.7)$ \\
\hline$\lambda$ & $293(48.7)$ \\
\hline$k+\lambda$ & $4(0.7)$ \\
\hline Free light-chain ratio & $440^{c}$ \\
\hline Normal (0.26-1.65) & $342(77.7)$ \\
\hline Abnormal & $98(22.3)$ \\
\hline M protein level and free light-chain ratio & $440^{c}$ \\
\hline $\begin{array}{l}\text { M protein }<15 \mathrm{~g} / \mathrm{L} \text { and free light-chain } \\
\text { ratio normal }\end{array}$ & $340(77.3)$ \\
\hline M protein $\geq 15 \mathrm{~g} / \mathrm{L}$ only & $2(0.4)$ \\
\hline Free light-chain ratio abnormal only & $90(20.5)$ \\
\hline Both abnormal & $8(1.8)$ \\
\hline $\begin{array}{l}M \text { isotype, } M \text { protein level, and free light- } \\
\text { chain ratio }\end{array}$ & $440^{c}$ \\
\hline $\begin{array}{l}\text { IgG type, M protein }<15 \mathrm{~g} / \mathrm{L} \text {, and free light- } \\
\text { chain ratio normal }\end{array}$ & $251(57.1)$ \\
\hline Any 1 abnormal & $137(31.1)$ \\
\hline Any 2 abnormal & $51(11.6)$ \\
\hline Any 3 abnormal & $1(0.2)$ \\
\hline $\begin{array}{l}\text { Reduced concentration of uninvolved } \\
\text { immunoglobulins }\end{array}$ & $313^{d}$ \\
\hline 0 & $265(84.7)$ \\
\hline 1 & $39(12.5)$ \\
\hline 2 & $8(2.5)$ \\
\hline 3 & $1(0.3)$ \\
\hline Type of M protein (ulFE) & $165^{\mathrm{e}}$ \\
\hline k & $21(12.7)$ \\
\hline$\lambda$ & $11(6.7)$ \\
\hline $\mathrm{M}$ protein & $4(2.4)$ \\
\hline $\mathrm{K}+\mathrm{M}$ protein & $1(0.6)$ \\
\hline
\end{tabular}

${ }^{a}$ The percentage was calculated as the number of patients with MGUS divided by the number who were tested.

beople who had tested sIFE were studied.

'People who had tested free light ratio (FLC) were studied.

dPeople who had tested immunoglobulins were studied.

ePeople who had tested uIFE were studied. Among them, 37 people had positive results.

studies showing that Asians have a lower incidence of MGUS compared with Whites. In the same age group (aged $\geq 50$ years), the prevalence of our study was lower than prior MGUS prevalences in Olmsted County, USA ${ }^{3}$, Germany $^{10}$, France ${ }^{11}$, and even the Asian country Japan ${ }^{12}$ (Supplementary Table 3). The considerable disparities may derive from differences in ethnicity, sampling strategies, detection techniques, environmental exposures, and lifestyles. We also noticed that the prevalence of
MGUS increases with advancing age and men had a significantly higher prevalence of MGUS than women.

The concentration of monoclonal protein in our study was extremely small (median, $1.5 \mathrm{~g} / \mathrm{L}$ ); $24.8 \%$ of patients had unmeasurable protein concentrations, and only $1.9 \%$ had a concentration of $\geq 15 \mathrm{~g} / \mathrm{L}$. However, the median concentration of monoclonal protein was $5 \mathrm{~g} / \mathrm{L}$ in the Olmsted County study, and $19.9 \%$ of participants had monoclonal immunoglobulin $>15 \mathrm{~g} / \mathrm{L}^{3}$. Because our study enrolled more young participants who may have a shorter underlying course and lower disease burden, it is reasonable to interpret these varied $M$ protein levels by age group. The most common immunoglobulin type in MGUS identified in our study was IgG (65.8\%), which was also observed in previous studies, ${ }^{3,11-13}$.

According to Mayo Clinic model for risk of progres$\operatorname{sion}^{14}$, more than half of MGUS patients $(57 \%)$ in our study had low-risk MGUS (M protein level $<15 \mathrm{~g} / \mathrm{L}$, IgG type, normal FLC ratio), and $43 \%$ had intermediate risk (any one or two factors abnormal), and only one patient $(0.23 \%)$ was prone to high risk of progression (three factors abnormal). However, among the Minnesotan MGUS patients, low-risk MGUS accounted for $38.8 \%$, intermediate risk for $56.4 \%$, and the high risk for $4.8 \%{ }^{1}$. Recent studies demonstrated that suppression of uninvolved immunoglobulins defined by heavy/light-chain pair suppression is a risk factor for progression of MGUS ${ }^{15}$. Nevertheless, among patients detected for serum immunoglobulins, most (84\%) had normal uninvolved immunoglobulins, in line with previous studies ${ }^{3}$.

In summary, our population-based study provides ageand sex-specific prevalences of MGUS in a geographically defined population that is generally representative of the Chinese healthy population. The differences in prevalence and risk factors of MGUS among various ethnic populations remain to be further explored.

\section{Acknowledgements}

This study is supported by the National Natural Science Foundation of China (Grant No. 81570195, to J.L.), Beijing Natural Science Foundation (Grant No. 7182128, to J.L.), The Capital Health Research and Development of Special Fund (Grant No. 2018-2-4015, to J.L.), the CAMS Innovation Fund for Medical Sciences (Grant No. 2016-12M-1-002, to J.L.), and The National Key Research and Development Program of China (Grant No. 2016YFC0901503, to J.L.).

\section{Author details \\ 'Department of Clinical Laboratory, Peking Union Medical College Hospital, Chinese Academy of Medical Sciences and Peking Union Medical College, Beijing, China. ${ }^{2}$ Department of Hematology, Peking Union Medical College Hospital, Chinese Academy of Medical Sciences and Peking Union Medical College, Beijing, China. ${ }^{3}$ Medical Research Center, Peking Union Medical College Hospital, Chinese Academy of Medical Sciences and Peking Union Medical College, Beijing, China. ${ }^{4}$ Department of Health Medicine, Peking Union Medical College Hospital, Chinese Academy of Medical Sciences and Peking Union Medical College, Beijing, China}

\section{Author contributions}

T.-d.X., J.L., and W.S. designed the research. J.-h.H., J.-n.W., and Y.-I.Z. collected, analyzed, and interpreted the data; J.-n.W., Y.-I.Z. X.-x.C., and J.L. wrote the 
manuscript. J.-H.H., W.S., and D.-b.Z. participated in data interpretation, reviewed the manuscript, and provided comments. All authors reviewed and approved the final manuscript.

\section{Conflict of interest}

The authors declare that they have no conflict of interest.

\section{Publisher's note}

Springer Nature remains neutral with regard to jurisdictional claims in published maps and institutional affiliations.

Supplementary Information accompanies this paper at (https://doi.org/ 10.1038/s41408-020-0303-8).

Received: 23 December 2019 Revised: 17 February 2020 Accepted: 27 February 2020

Published online: 09 March 2020

\section{References}

1. Kyle, R. A. et al. Long-term follow-up of monoclonal gammopathy of undetermined significance. N. Engl. J. Med. 378, 241-249 (2018).

2. Wadhera, R. K. \& Rajkumar, S. V. Prevalence of monoclonal gammopathy of undetermined significance: a systematic review. Mayo Clin. Proc. 85, 933-942 (2010).

3. Kyle, R. A. et al. Prevalence of monoclonal gammopathy of undetermined significance. N. Engl. J. Med. 354, 1362-1369 (2006).

4. Landgren, $\mathrm{O}$. et al. Racial disparities in the prevalence of monoclonal gammopathies: a population-based study of 12482 persons from the national health and nutritional examination survey. Leukemia 28, 1537-1542 (2014).
5. Watanaboonyongcharoen, P., Nakorn, T. N., Rojnuckarin, P., Lawasut, P. \& Intragumtornchai, T. Prevalence of monoclonal gammopathy of undetermined significance in Thailand. Int. J. Hematol. 95, 176-181 (2012).

6. Lee, Y. G. et al. Five-year follow-up study of monoclonal gammopathy of undetermined significance in a Korean elderly urban cohort. Cancer Res. Treat. 47, 215-220 (2014).

7. Peter, S. W. et al. MGUS prevalence in an ethnically Chinese population in Hong Kong. Blood 121, 2363-2364 (2013).

8. Vincent, S. R. et al. International Myeloma Working Group updated criteria for the diagnosis of multiple myeloma. Lancet Oncol. 15, e538-e548 (2014).

9. Katzmann, J. A. et al. Serum reference intervals and diagnostic ranges for free $\mathrm{K}$ and free $\lambda$ immunoglobulin light chains: relative sensitivity for detection of monoclonal light chains. Clin. Chem. 48, 1437-1444 (2002).

10. Eisele, L. et al. Prevalence and progression of monoclonal gammopathy of undetermined significance and light-chain MGUS in Germany. Ann. Hematol. 91, 243-248 (2012)

11. Lecluse, $Y$. et al. Prevalence of monoclonal gammopathy of undetermined significance (MGUS) among farmers involved in open field farming and/or cattle breading in France. Leuk. Lymphoma 57, 1727-1730 (2016).

12. Iwanaga, M., Tagawa, M., Tsukasaki, K., Kamihira, S. \& Tomonaga, M. Prevalence of monoclonal gammopathy of undetermined significance: study of 52,802 persons in Nagasaki City, Japan. Mayo Clin. Proc. 82, 1474-1479 (2007).

13. Cabrera, Q. et al. Epidemiology of monoclonal gammopathy of undetermined significance (MGUS): the experience from the specialized registry of hematologic malignancies of Basse-Normandie (France). Cancer Epidemiol. 38, 354-356 (2014).

14. Kyle, R. et al. Monoclonal gammopathy of undetermined significance (MGUS) and smoldering (asymptomatic) multiple myeloma: IMWG consensus perspectives risk factors for progression and guidelines for monitoring and management. Leukemia 24, 1121-1127 (2010).

15. Katzmann, J. A. et al. Suppression of uninvolved immunoglobulins defined by heavy/light chain pair suppression is a risk factor for progression of MGUS. Leukemia 27, 208-212 (2013). 